\title{
Synchronous Primary Adenocarcinoma of the Lung and Leiomyosarcoma of the Small Intestine
}

\author{
Yuji Takemoto, Masaaki Kawahara, Satoru Yamamoto*, Keiji Iuchi**, Takashi Mori**, Einosuke Ueda, \\ Tessei TsuchiYama and Kiyoyuki Furuse
}

\begin{abstract}
The occurrence of synchronous epithelial cancer of the lung and leiomyosarcoma of the small intestine is rare. We report here the case of a 62 -year-old man with adenocarcinoma of the lung in clinical stage IIIB (T4NOMO). After two courses of chemotherapy (cisplatin, $80 \mathrm{mg} / \mathrm{m}^{2}$ and $\mathrm{mi}-$ tomycin $\mathrm{C}, 8 \mathrm{mg} / \mathrm{m}^{2}$ ) the patient developed symptoms of a small bowel obstruction. Palliative surgical resection was performed and a leiomyosarcoma of the small intestine was found and defined by an immunohistological study. The resection ameliorated the patient's symptoms. The patient died of disseminated adenocarcinoma 26 months following chemotherapy.

(Internal Medicine 39: 655-658, 2000)
\end{abstract}

Key words: leiomyosarcoma of the small intestine, pulmonary adenocarcinoma, spindle cells of metastatic lung cancer, synchronous tumor

\section{Introduction}

Lung cancer is characterized by metastases to the brain, bone, liver, and adrenal glands. Metastasis of lung cancer to the small bowel occurs infrequently, but causes perforation, obstruction, malabsorption and/or hemorrhaging (1). The appearance of small intestinal lesions following pulmonary adenocarcinoma is mostly thought to be due to metastasis. Spindle cell variants of pulmonary adenocarcinoma in metastatic lesions have been reported. These reports concluded that the metastasis exclusively composed of spindle cells was misdiagnosed as leiomyosarcoma $(2,3)$. Small intestinal occlusion by metastatic tumors is treated conservatively (4). Primary smooth muscle tumors of the gastrointestinal tract are uncommon and diagnosed mostly as leiomyoma (5). Primary leiomyomas make up $71.8 \%$ of these tumors and are sometimes treated by excision (6). The percentage of tumors potentially cured by surgery and the 5-year survival rates of the leoimyosarcoma $(75 \%$ and $57 \%$, respectively) were higher than that of adenocarcinoma ( $42 \%$ and $10 \%$, respectively) (7). We present a case in which leiomyosarcoma was discovered while investigating an ileus found during a course of treatment for pulmonary adenocarcinoma. Synchronous leiomyosarcoma of the small bowl and pulmonary adenocarcinoma were confirmed by an immunohistochemical study. Surgical resection of leiomyosarcoma improved the patient's quality of life and was useful in decreasing various abdominal symptoms.

\section{Case Report}

In January 1996, the chest X-ray of a 62-year-old man smoker exhibited an abnormal mass shadow. The histology of the transbronchial tumor biopsy indicated papillary adenocarcinoma compatible with primary lung cancer (Fig. 1). A stage IIIB tumor, $22 \times 27 \mathrm{~mm}$ in size, was identified in the posterior segment of the upper right lung and was accompanied by pleural effusion, but not by mediastinal invasion on chest CT scan. Simultaneously, the patient sometimes complained of abdominal pain, and an abdominal X-ray in March 1996 showed multiple air-fluid levels in the small bowel. From the examination of the abdominal CT, the abdominal pain was thought to have been caused by paralytic ileus. Abdominal pain and ileus were ameliorated by conservative therapies: pharmacological treatment, nasogastric suction and intravenous fluid. After conservative therapies, the performance status of the patient was 1 (symptomatic; fully ambulatory) and chemotherapy for cancer was thought to be effective. The patient received two courses of combination chemotherapy (cisplatin, $80 \mathrm{mg} / \mathrm{m}^{2}$ and mitomycin $\mathrm{C}, 8 \mathrm{mg} / \mathrm{m}^{2}$ ). The patient's condition was systematically ameliorated. Severe abdominal pain and ileus developed in June 1996 and an obstructive mass in the small bowel was found. A single occlusive lesion in the small intestine was detected by

From the Department of Internal Medicine, National Kinki Central Hospital, Osaka, *the Department of Pathology, National Kinki Central Hospital, Osaka and

**the Department of Surgery, National Kinki Central Hospital, Osaka

Received for publication May 10, 1999: Accepted for publication February 9, 2000

Reprint requests should be addressed to Dr. Yuji Takemoto, the Department of Internal Medicine, National Kinki Central Hospital, 1180 Nagasonecho, Sakai, Osaka 591-8025 


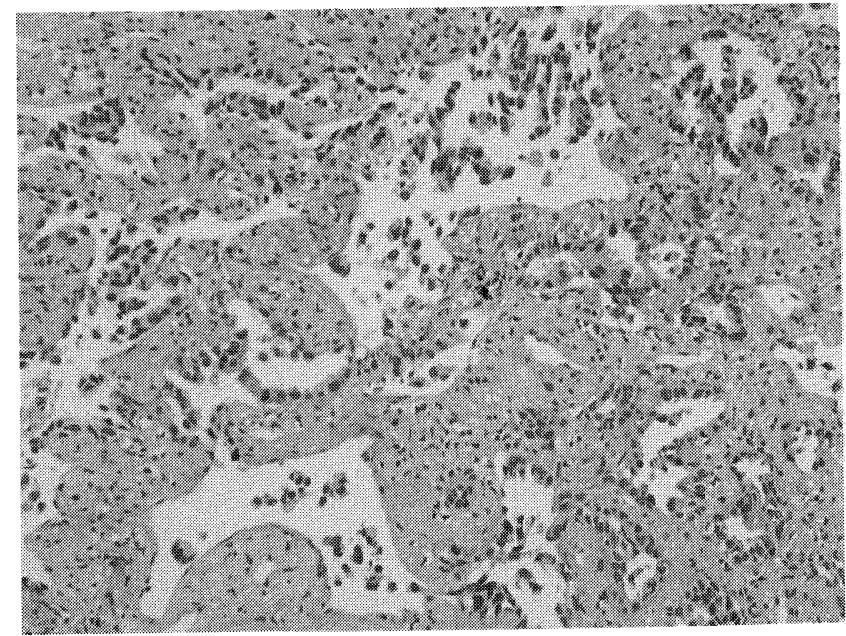

Figure 1. Histologic findings of the TBLB specimens, showing papillary adenocarcinoma in the fibrous parenchyma ( $\mathrm{HE}$ stain, $\times$ 100).

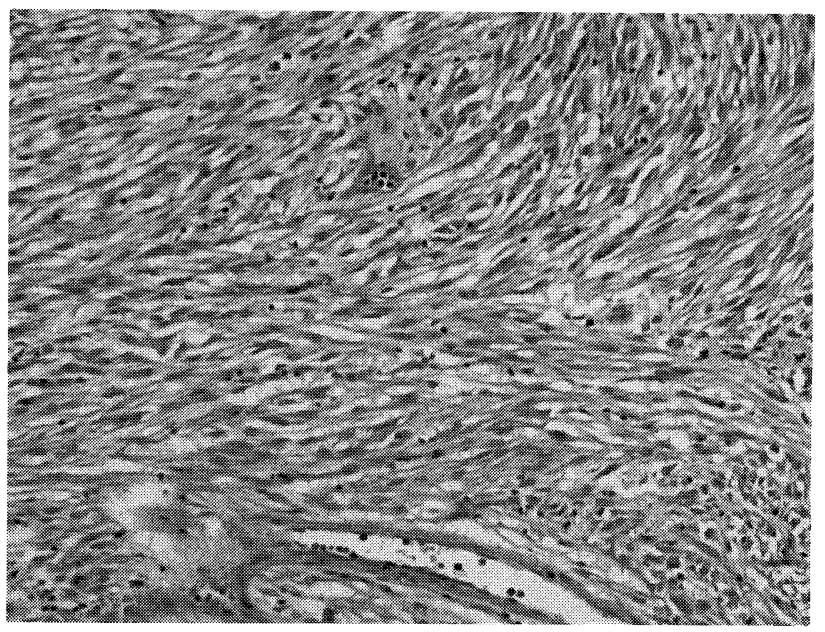

Figure 2. Histological findings of the excised tumor $(5.6 \times 5 \times 4$ cm) in the small intestine, showing spindle shape cells and non transitional zone (HE stain, $\times 100$ ).

preoperative study. Surgical excision was performed in July 1996. Microscopically, the tumor consisted of spindle-shape cells in an interlacing or fascicular pattern with some mitotic activity (Fig. 2). An immunohistochemical study was performed on the formalin-fixed, paraffin-embedded sections using the labelled streptavidin-biotin peroxidase method (Dako Japan, Kyoto). Carcino-embryonic antigen (CEA), keratin, vimentin, desmin, $\alpha$-smooth muscle actin and S-100 protein were applied for the immunohistochemical study (Dako Japan ). The tumor cells demonstrated a strong positive reaction to vimentin and $\alpha$-smooth muscle actin (Figs. 3, 4). However, there was a negative reaction for CEA, keratin, desmin, and S-100 pro-

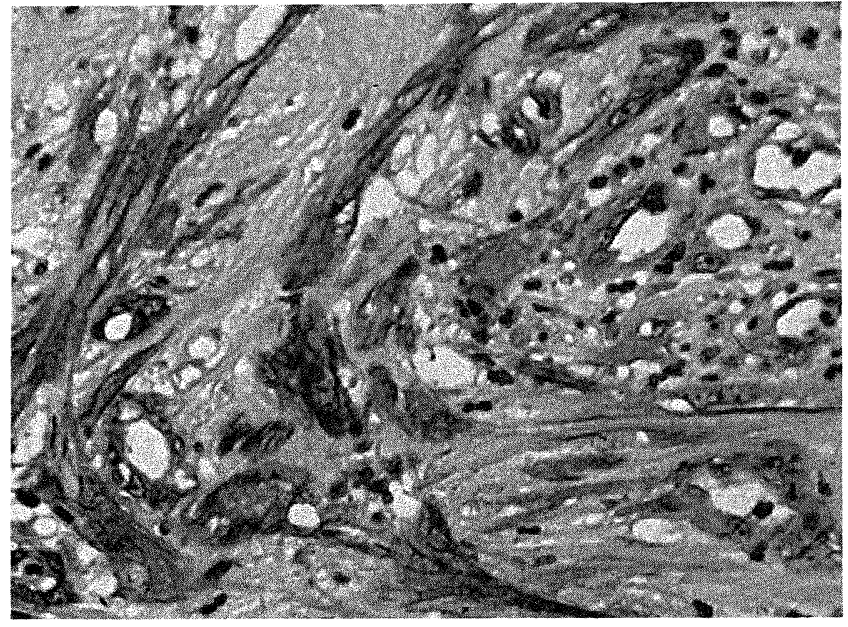

Figure 3. Immunohistochemical findings of a small intestinal tumor. Almost all of the tumor cells were positive for (alpha)-smooth muscle actin.

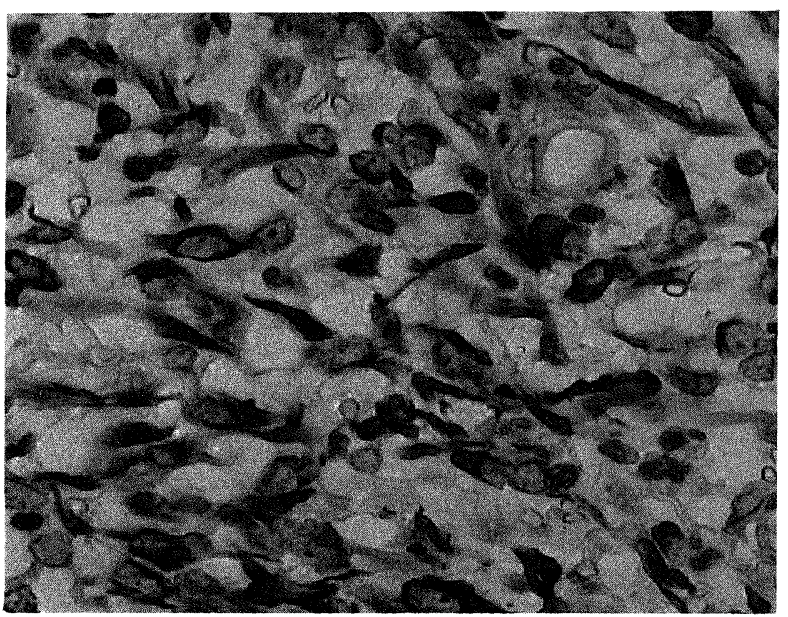

Figure 4. Immunohistochemical findings of a small intestinal tumor, spindle shape cells show a positive reaction for vimentin.

tein. Additionally, the transbronchial biopsy revealed that the tumor consisted of papillary adenocarcinoma without sarcomatous components. Immunohistochemically, there was a positive reaction to CEA and keratin but a negative reaction to the other mesenchymal markers (vimentin and $\alpha$-smooth muscle actin). No histological similarity was shown between the species of the lung and the intestinal tumor. Consequently, the tumor was diagnosed as leiomyosarcoma of the small intestine. After the operation the patient had no abdominal symptoms. Right pleural effusion due to the progression of lung cancer appeared in October 1996. The patient received two courses of second line combination chemotherapy (carboplatin and 
irinotecan) in September 1996. The second treatment produced no changes in the patient's condition, and thus picivanyl was administered intrapleurally. Pleural effusion disappeared and the patient underwent followup treatment in the outpatient clinic without any complaint for 7 months, but he experienced bilateral chest pain in September 1997. MRI showed metastasis from the seventh to tenth thoracic vertebral bones and a compression of the spinal cord in October 1997. The patient was readmitted to our hospital and radiotherapy $(40 \mathrm{~Gy})$ to the metastatic vertebral bone and spinal cord was given. Paraplegia did not develop. Brain metastasis of lung cancer developed in October 1997 and he died of lung cancer in April 1998. An autopsy could not be performed.

\section{Discussion}

Spindle cell proliferation of the small bowel following pulmonary adenocarcinoma is difficult to diagnose. Spindle cell formation has sometimes been shown in pulmonary blastoma, carcinosarcoma and mesothelioma $(7,8)$. Spindle cells themselves are composed of a variant of epithelial, mesenchymal, adenomatous or squamous components $(7,9)$. There are two reports concerning spindle cells of pulmonary adenocarcinoma. The first is a case of pulmonary adenocarcinoma with sarcomatous elements initially manifested as a submucosal tumor of the stomach (3). The other is a case of pulmonary adenocarcinoma with spindle cell variant in a transverse lesion of the spinal cord (2). Extra-pulmonary lesions in these cases were initially diagnosed as leiomyosarcoma and autopsy disclosed spindle cell variants of pulmonary adenocarcinoma. According to Drlicek et al, there are four differential diagnostic possibilities for cases such as the present case: a) two different primary tumors, an adenocarcinoma of the lung and a small intestinal leiomyosarcoma, b) adenosquamous carcinoma of the lung with a spindle differentiated squamous part, c) biphasic mesothelioma and d) adenocarcinoma with a spindle cell component (2). Confirmation by an immunohistochemical study and the presence of a transitional zone is important for diagnosis of primary or metastatic spindle cell. An immunohistochemical study discloses the origin of spindle cells. Keratin and desmin are positive markers for epithelial and mesenchymal components. Adenomatous components express keratin, CEA and S100 protein. The positive reaction of vimentin and/or $\alpha$-smooth muscle actin is observed in myogenic components. In this case the zone of transition from adenomatous to spindle cells part could not be detected. Spindle cell tumor of the small intestine was not derived from pulmonary adenocarcinoma.

We have optional treatments for the management of bowel obstruction in advanced cancer patients: surgery, nasogastric suction and intravenous fluids, pharmacological treatment and percutaneous gastrectomy. There are many debates concerning the surgical treatment of bowel obstruction in advanced and terminal patients (4). Small bowel metastasis will always signify poor prognosis (10); the median survival after diagnosis for this problem is 4 weeks (limits: 1 to $20+$ weeks) (11). Multiple obstructive lesions, previous palliative surgery and poor performance status ( $<30$ on the Karnofsky scale) are contraindications for surgery, and palliative care such as the administration of morphine or metoclopramide is preferable (4). According to different authors, benign obstructions are present in $3 \%$ to $48 \%$ cases of advanced cancer. The impossibility of resection of these obstructions is mostly due to the presence of extensive tumors, multiple or partial obstruction, or the impossibility of surgical correction (4). Surgery does not always ameliorate obstructive symptoms and, in $9 \%$ to $80 \%$ of cases, it can lead to further complications (4). Also surgery is not attempted in cases where there is no clear indication that the patient will survive at least 60 days after the operation with no further complications $(1,12)$. The present patient had been treated with nasogastric suction and intravenous fluid prior to surgical treatment. The ineffectiveness of these conservative therapies, good performance status of the patient, single obstructive locus and no metastasis of the tumor lead to the decision for surgical treatment. Small bowel tumors are not so frequent and it is difficult to conclude the character of the tumor prior to surgery. Surgical treatment must only be attempted after careful consideration, based on the patient's performance status, metastasis and prognosis. The patient survived 14 months following resection of leiomyosarcoma and died of lung cancer 26 months following the initiation of chemotherapy. The five-year survival rate for stage IIIA and IIIB lung cancer are $13 \%$ and $5 \%$ (13) and chemotherapy for the patient was effective in improving the prognosis. It is important to diagnose whether metastasis has occurred or not, as the prognosis between a patient with synchronous leiomyosarcoma and lung cancer and a patient with stage IV of lung cancer differ greatly.

In conclusion, an immunohistological study and the presence of a transitional zone in histology were necessary for the differential diagnosis of spindle cells of the small intestine from pulmonary adenocarcinoma. We found that a patient diagnosed as having synchronous advanced lung cancer and small bowel leiomyosarcoma survived longer than cases with small bowel metastasis of lung cancer. Additionally, the excision of the small bowel leiomyosarcoma was beneficial to the patient's quality of life in the present.

Acknowledgements: we are grateful to Mr. Robert Condon for his proofreading and ameudment of this English virsion of this manuscript.

\section{References}

1) Mcneill PM, Wagman LD, Neifeld JP. Small bowel metastases from primary carcinoma of the lung. Cancer 59: 1486-1489, 1987.

2) Drlicek M, Liszka, U, Machacek E, Grisold W, Lintner F. Spindle cell variant of pulmonary adenocarcinoma. Pathol Res Pract 189: 586-590, 1993.

3) Fukuda T, Ohnishi Y, Katagiri J, Ohnuki K, Tachikawa S. A case with pulmonarycarcinoma with sarcomatous elements initially manifested as a submucosal tumor of the stomach. Acta Pathol Jpn 42: 454-459, 1992.

4) Ripamonti C, Conno F, Ventafridda V, Rossi B, Baines MJ. Management of bowel obstruction in advanced and terminal cancer patients. Ann Onco 4: 15-21, 1993.

5) Stavorovsky M, Morag B, Starvorosky H, Papo J. Smooth muscle tumors of the alimentary tract. J Surg Onco 22: 109-114, 1983. 


\section{TAKEMOTO et al}

6) Spiliotis J, Scopa CD, Kyriakopoulou D, et al. Smooth muscle tumors of the gastrointestinal tract. A 10-year experience. Eur J Surg Onco 18: 580$584,1992$.

7) Kusumoto H, Takahashi I, Yoshida M, et al. Primary malignant tumors of the small intestine: analysis of 40 Japanese patients. J Surg Oncol 50: 139-143, 1992.

8) World Health Organization (1981) International histological classification of tumors. No. 1. Histological typing of lung tumors. (Second edition) Genova: World Health Organization, 33-34.

9) Yousem SA, Wick MR, Randhawa P, Manivel JC. Pulmonary blastoma: an immunohistochemical analysis with comparison with fetal lung in its pseudoglandular stage Am J Cli Pathol 93: 167-175, 1990.
10) Santeusanio G, Pascal RR, Bisceglia M, Costantino AM, Bosman C. Metaplastic breast carcinoma with epithelial phenotype of pseudosarcomatous components Arch Pathol Lab Med 112: 82-85, 1988.

11) Centeno Cortes C, Borau Clavero MJ, Sanz Rubiales A, Lopez-lara Martin F. Intestinal bleeding in disseminated non -small cell lung cancer. Lung Cancer 18: 101-105, 1997.

12) Castaldo TW, Petrilli ES, Ballon SC Lagasse LD. Intestinal operations in the patients with ovarian carcinoma. Am J Obstet Gyneco 139: 80-84, 1981.

13) Mountain CF. Revisions in the International system for staging lung cancer. Chest 111: 1710-1717, 1997. 